\title{
Rapid Response to Certolizumab Pegol in Hidradenitis Suppurativa: A Case Report
}

\author{
Pelin Esme Gulsen Akoglu Ercan Caliskan \\ Department of Dermatology and Venereology, Gulhane School of Medicine, University of Health Sciences, Ankara, Turkey
}

\section{Established Facts}

- Hidradenitis suppurativa (HS) is a chronic inflammatory skin disease of hair follicles with a highly painful and destructive disease course.

- The role of immunomodulation in the clinical management of HS is becoming increasingly important with the advances in its pathogenesis.

- Adalimumab is the only FDA-approved biologic agent for treatment of HS; however, clinical management of some HS patients under adalimumab is still quite challenging.

- Certolizumab is a humanized antigen-binding fragment of a monoclonal antibody which binds to TNF-alpha, and it is approved for only plaque psoriasis in dermatology.

\section{Novel Insights}

- Certolizumab pegol is likely to be a promising effective agent for HS, especially in challenging cases.

\section{Keywords}

Hidradenitis suppurativa · Medical dermatology · Biologics · Certolizumab pegol $\cdot$ Secukinumab

\begin{abstract}
Introduction: Hidradenitis suppurativa (HS) is a chronic inflammatory skin disease of hair follicles which usually affects patients' quality of life negatively. Although there are various therapeutic approaches including topical and systemic antibiotics, retinoids, biological agents, and also surgical modalities, there is no curative treatment option and clinical management of HS is still challenging. Case Presentation: Herein, we present a 43 year-old man with HS who had used different treatments in 15 years. The patient showed improvement initially with adalimumab, then exhibited sec-
\end{abstract}

ondary unresponsiveness after 18 months. While worsening of HS was recorded due to increased discharge under secukinumab, no additional benefit was observed with methotrexate. He was not willing to undergo surgical procedures due to previous surgical experience. Finally, certolizumab pegol has been started to patient with similar dosing to psoriasis patients. Sartorius, 10-point visual analogue scale (VAS), and dermatological life quality index (DLQI) scores regressed from 171 to $105,9 / 10$ to $3 / 10$, and 27 to 19 after 3 months with certolizumab pegol treatment. Discussion/Conclusion: Certolizumab pegol is a humanized antigen-binding fragment of a monoclonal antibody which binds to TNF-alpha. However, certolizumab pegol is only approved for plaque psoriasis in dermatology; it is likely to be a promising effective agent for $\mathrm{HS}$, especially in challenging cases.

$\begin{aligned} & \text { karger@karger.com } \\ & \text { www.karger.com/sad }\end{aligned}$
Karger $\%$ 2020 S. Karger AG, Basel




\section{Introduction}

Hidradenitis suppurativa (HS) is a chronic, inflammatory, recurrent, and debilitating skin disease of the hair follicles, which is usually difficult to manage [1]. Herein, we present a challenging case in which rapid and meaningful clinical improvement could be achieved with certolizumab pegol treatment.

\section{Case Presentation}

A 43-year-old man who had been diagnosed as having HS 15 years ago, had used different treatment options including numerous topical and systemic antibiotics and retinoids. He was operated for pilonidal sinus and Fournier's gangrene. Bilateral axillary areas, intergluteal sulcus and especially the right glutea were very severely involved as Hurley stage 3 . Despite rapid response with adalimumab, his complaints gradually started to increase after 18 months. Therefore, we decided to switch the treatment to
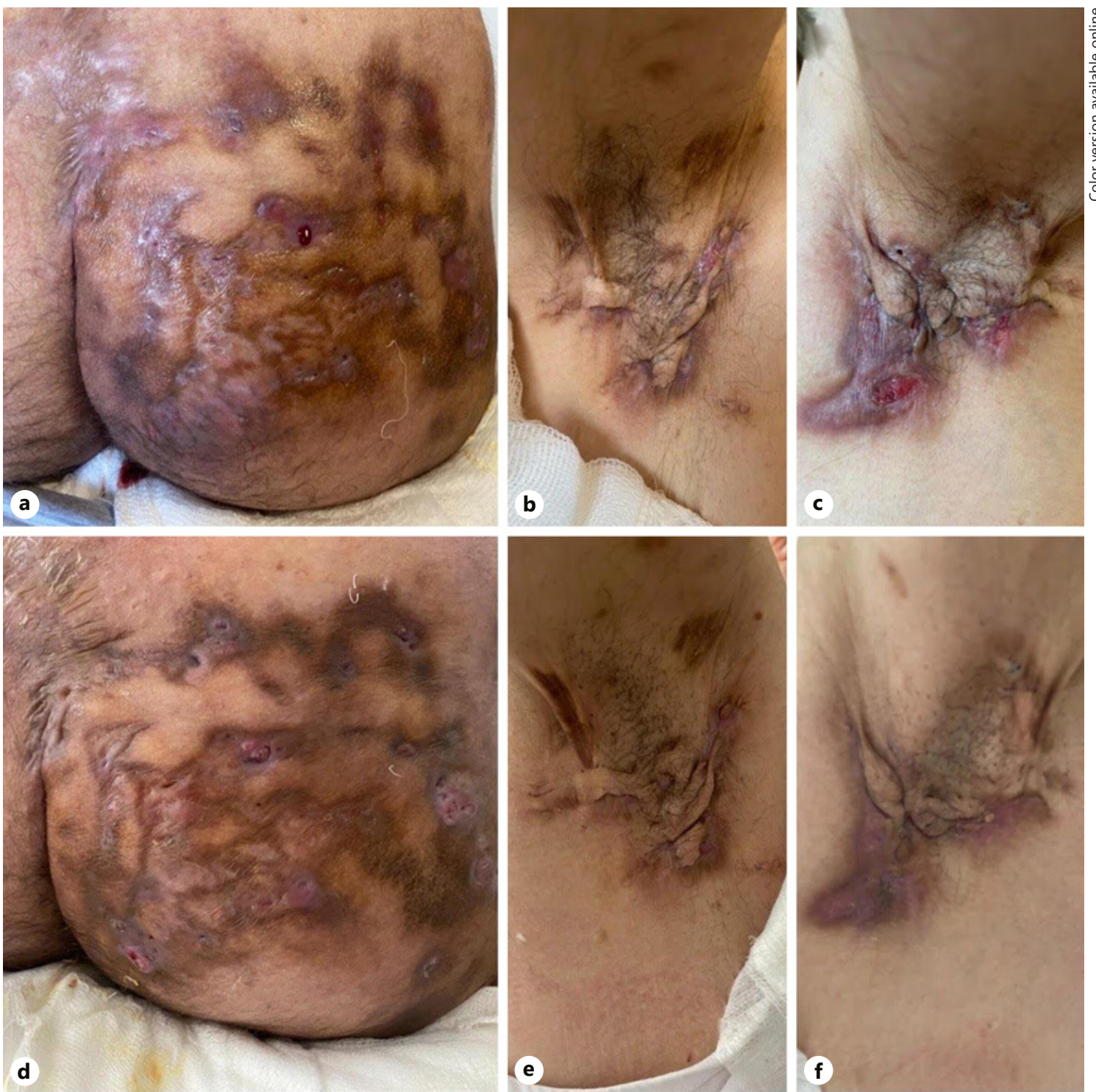

Fig. 1. Photographs of baseline (a-c) and after treatment with 3-month certolizumab pegol (d-f). Diminished inflammation and discharge on the right glutea (a-d), right axilla (b-e), and left axilla (c-f) are seen after 3-month certolizumab pegol treatment. 
secukinumab therapy. However, HS lesions worsened and especially the discharge on his right buttock increased at 10th week of secukinumab due to "Candida glabrata" overgrowth which was evaluated by microbial cultures. Administration of anti-candidal treatments and cessation of the secukinumab could achieve negative culture results and improvement. Treatment plan arranged to methotrexate; however, no sufficient response could be obtained at the end of 3 months. Therefore, the patient was recommended to undergo a surgical treatment for right gluteal region, but he has refused. Finally, certolizumab pegol has been started to patient with similar dosing to psoriasis patients: $400 \mathrm{mg}$ in every other week [2]. Although the patient was informed about possible risks of immunosuppressive agents during coronavirus disease 2019 pandemic, he insisted to continue the current treatment owing to $80 \%$ decrease in discharge and pain scores at the end of the 1 st month of certolizumab treatment and a signed informed consent was obtained from the patient. HS Clinical Response (HiSCR) [3] was achieved at 12th week. Sartorius, 10-point visual analogue scale (VAS) and dermatological life quality index (DLQI) scores regressed from 171 to $105,9 / 10$ to $3 / 10$, and 27 to 19 after 3 months with certolizumab pegol treatment. Clinical improvement of the patient under 3-month certolizumab pegol treatment is shown in Figure 1.

\section{Discussion}

Certolizumab is a humanized antigen-binding fragment of a monoclonal antibody which binds to TNF-alpha. It is approved for only plaque psoriasis in dermatology [2]. Although complex pathogenesis of HS has not been fully understood, the role of abundant expression of pro-inflammatory cytokines especially TNF-alpha, IL-12, IL-24, IL-17, and blockade of these cytokines for clinical management is quite obvious [4]. The only approved biologic treatment for HS is adalimumab (strength of recommendation is A), with HiSCR responses restricted to 42 and $59 \%$ of all HS patients in PIONEER 1 and PIONEER 2 phase 3 trials, respectively [3]. While infliximab (strength of recommendation is $\mathrm{B}$ ) is recommended to patients who were unresponsive to adalimumab, limited data do not support the use of etanercept and placebo-controlled dose ranging studies are needed for the optimal dosing of anakinra and ustekinumab $[1,5]$. Currently, secukinum$\mathrm{ab}$ offers very promising results due to the regulation of Thelper 17 imbalance in HS [6, 7]. Secukinumab is known to cause an increase in the occurrence of Candida infections with blockade of IL-17 which is known as a major cytokine for protection against cutaneous and mucosal Candida infections [8]. However, candida infections are well described during secukinumab treatment in psoriasis with a prevalence between 1.11 and $1.7 \%$, and there are still no data about HS $[8,9]$.

60

Skin Appendage Disord 2021;7:58-61 DOI: $10.1159 / 000511284$
Successful treatment with certolizumab pegol of a pregnant patient with both HS and psoriasis was mentioned in the literature; however, details were not reported [10]. In the current report, we fully documented a patient with HS who showed rapid and significant response with certolizumab pegol treatment.

\section{Conclusion}

Although future long-term large scaled studies and observations are needed, certolizumab pegol is likely to be a promising effective agent for HS, especially in challenging cases.

\section{Statement of Ethics}

Written informed consent was obtained from the patient for publication (including publication of images).

\section{Conflict of Interest Statement}

The authors have no conflicts of interest to declare.

\section{Funding Sources}

The authors did not receive any funding.

\section{Author Contributions}

Pelin Esme: drafting the work, substantial contributions to the conception, and design of the work, or the acquisition, analysis, or interpretation of data for the work. Gulsen Akoglu: revising it critically for important intellectual content and final approval of the version to be published. Ercan Caliskan: revising it critically for important intellectual content and final approval of the version to be published.

References

1 Alikhan A, Sayed C, Alavi A, Alhusayen R, Brassard A, Burkhart C, et al. North American clinical management guidelines for hidradenitis suppurativa: a publication from the United States and Canadian Hidradenitis Suppurativa Foundations: part I: diagnosis, evaluation, and the use of complementary and procedural management. J Am Acad Dermatol. 2019;81(1):76-90.

2 Menter A, Strober BE, Kaplan DH, Kivelevitch D, Prater EF, Stoff B, et al. Joint AADNPF guidelines of care for the management and treatment of psoriasis with biologics. J Am Acad Dermatol. 2019;80(4):1029-72.

Esme/Akoglu/Caliskan 
3 Kimball AB, Okun MM, Williams DA, Gottlieb AB, Papp KA, Zouboulis CC, et al. Two phase 3 trials of adalimumab for hidradenitis suppurativa. N Engl J Med. 2016;375(5):42234.

4 Zouboulis CC, Desai N, Emtestam L, Hunger $\mathrm{RE}$, Ioannides D, Juhász I, et al. European S1 guideline for the treatment of hidradenitis suppurativa/acne inversa. J Eur Acad Dermatol Venereol. 2015;29(4):619-44.

5 Ingram JR, Collier F, Brown D, Burton T, Burton J, Chin MF, et al. British Association of Dermatologists guidelines for the management of hidradenitis suppurativa (acne inversa) 2018. Br J Dermatol. 2019;180(5):100917.
6 Casseres RG, Prussick L, Zancanaro P, Rothstein B, Joshipura D, Saraiya A, et al. Secukinumab in the treatment of moderateto-severe hidradenitis suppurativa: results of an open-label trial. J Am Acad Dermatol. 2020;82(6):1524-26.

7 Prussick L, Rothstein B, Joshipura D, Saraiya A, Turkowski Y, Abdat R, et al. Open-label, investigator-initiated, single-site exploratory trial evaluating secukinumab, an anti-interleukin-17A monoclonal antibody, for patients with moderate-to-severe hidradenitis suppurativa. Br J Dermatol. 2019;181(3):60911.
8 Saunte DM, Mrowietz U, Puig L, Zachariae C. Candida infections in patients with psoriasis and psoriatic arthritis treated with interleukin-17 inhibitors and their practical management. Br J Dermatol. 2017;177(1):47-62.

9 van de Kerkhof PC, Griffiths CE, Reich K, Leonardi CL, Blauvelt A, Tsai TF, et al. Secukinumab long-term safety experience: a pooled analysis of 10 phase II and III clinical studies in patients with moderate to severe plaque psoriasis. J Am Acad Dermatol. 2016; 75(1):83-e4.

10 Porter ML, Golbari NM, Lockwood SJ, Kimball $\mathrm{AB}$. Overview and update on biologic therapy for moderate-to-severe hidradenitis suppurativa. Semin Cutan Med Surg. 2018; 37(3):182-9. 